\title{
HUMAN-WHAT INTERACTION? UNDERSTANDING USER SOURCE ORIENTATION
}

\author{
Jacob Solomon and Rick Wash \\ Department of Media and Information \\ Michigan State University
}

\begin{abstract}
Interaction with internet-connected computing devices involves interaction with many distinct agents or sources simultaneously. Hardware, operating systems, web browsers, networking devices, and servers all influence the user experience, as do the engineers and programmers who designed them, the companies or organizations that have developed the systems, other users on the web, and various third-parties such as advertisers. We argue that users cannot be simultaneously engaged towards all of these sources of interaction, and instead must orient themselves to only a subset at any given time. We propose a model of source orientation based on literature from psychology, communications and human-computer interaction. This model describes how users select their source orientation when interacting with computers. We also present examples of how this model can be applied to promote usability in computing systems.
\end{abstract}

\section{INTRODUCTION}

When people interact with computers, they are interacting with several different entities or sources of behavior simultaneously. Even a basic interactive event which may appear to involve only one person can actually involve several other people or autonomous entities. For example, when conducting a web search, there are several distinct entities with which the user is interacting in some way. On one level, the user is interacting with a physical computer by viewing information on a monitor and typing the characters of the query onto a keyboard. On another level, the user is focusing the interaction on a specific aspect of the computer, a web browser. On yet another level, the user is interacting with another computer, or several other computers, which are acting as servers that execute the actual search. The user is also interacting with the company, i.e. Google or Yahoo, that runs the servers to which the web search has been requested. Programmers and designers have also carefully crafted the user experience for the website, the web browser, the operating system, and other software which is involved in the act of making a web search. When the user makes a query, he or she is interacting with those people as well. The user is also interacting with millions of others on the Internet who have created content or websites that will be retrieved in the search or have influenced the algorithms used to determine keyword relevance.

Any entity that influences an interaction with a computer is a source. Sometimes, it is not apparent which source (or sources) has influenced what the user sees or experiences. This makes human-computer interaction an interesting context in which people at any given moment may be only partially aware or cognizant of all the sources with which they interact. At any given moment, users may only be focusing their interaction on one of these sources or a small subset. And users may behave differently depending on which of these sources they are focused on at any given moment.

In this paper we discuss the human factors implications of a theoretical concept called source orientation. Source orientation is the psychological locus of a user's interaction in a mediated environment at a given time (Sundar \& Nass, 2000). Put more simply, a user's source orientation is the source (computer, other user, programmer, organization etc.) that the user considers to be the focus of the interaction. We argue here that source orientation can influence how people think about and use computers, particularly in online or networked environments. We propose a theoretical model that describes how users select, maintain or change their source orientation. We draw on important related concepts and findings from psychology, communications, and humancomputer interaction to build this model, and demonstrate how it can contribute to building user-centered technologies.

First we will discuss several bodies of literature that relate to and inform our understanding of source orientation. We will then integrate this work into a theoretical model of source orientation. Finally, we discuss some applications of source orientation to human factors and to user-centered design.

\section{REVIEW}

Source orientation has primarily been studied within the Computers-Are-Social-Actors paradigm (CASA) (Nass \& Moon, 2000). CASA is a series of experiments that replicate social psychology experiments, but replace some human element of the experiment with a computer. These studies show that computer users will be polite to computers, apply gender stereotypes, reciprocate favors, and other social responses that appear inappropriate for a computer. One possible explanation for these findings is that computer users have a source orientation to the computer's programmer or other humans "behind" the computer, rather than towards the computer itself. This would make such social responses seemingly appropriate.

CASA research, however, has found that most computer users do not think at all about the people who programmed it (Nass \& Moon, 2000), and that there is variation in source orientation among computer users (Hoffmann et al., 2009). Therefore, source orientation does not explain social responses to computers. Some research has induced users with a source 
orientation towards a computer's programmers or towards other distant agents (Sundar \& Nass, 2000; Tourangeau et al., 2003; Eckles et al., 2009; Shechtman \& Horowitz, 2003). This research has found that users may behave differently when they are oriented towards some sources than towards others.

However, this research has not established a systematic explanation of these differences. Therefore, we have reviewed several related concepts to develop an understanding of how source orientation might work. Below, we have summarized some important findings from this review.

\section{Attention}

There are two principal processes that govern the allocation of attention (Ashcraft, 2006). Exogenous or bottomup processing allocates attention to sensory stimuli in the environment. This process can be automatic, and is influenced by the salience of the stimuli. Endogenous or top-down processing allocates attention according to an individual's motivations, experiences, or other intrinsic qualities. These processes often compete for limited attention resources, as evidenced by a classic experiment in which $50 \%$ of subjects fail to notice a gorilla standing in the middle of a screen because they are intensely focused on completing a goal of counting the number of times a basketball is passed between people on the screen (Chabris \& Simons, 2009).

Some sources, such as the computer screen and its contents, are more immediate to a user and can therefore attract attention through bottom-up processing of sensory stimuli. Other sources, such as programmers or remote servers, are less immediate and therefore require some topdown processing by the user in order to attract attention. Since top-down processing requires a clear and voluntary motivation to attend to an object, orientation towards less immediate sources will likely only occur when such a motivation exists.

\section{Attribution Theory}

Making attributions of cause and effect may affect source orientation. For example, will a Facebook user attribute seeing a certain post on their newsfeed to Facebook's algorithm, or to their friend that posted it? And does that attribution influence who the user believes is the source of their interaction with the site? If so, he or she may behave differently depending on which attribution has been made.

Attribution theory seeks to explain how people determine causes and effects of events. This concept is important to understanding source orientation because many potential sources in an interaction may be hidden or not immediate, such as the programmer of a website or the network wi-fi router that enables connection. A lack of transparency of all sources may make it difficult for users to determine which source has had what effect in their interaction.

Factors of motivation, cognition, context, arousal, and intentionality all contribute to the process of attributing causality (see Kelley and Michela (1980) for a review). They argue that certain characteristics of information (such as its distinctiveness and consensus with the views and understandings of others), as well as motivational factors, strongly influence the ways that people make attributions.
There are also several biases in how people make attributions, such as underestimating the influence of situational factors in an outcome (Gilbert \& Malone, 1995) and overly attributing negative outcomes to external factors (Morewedge, 2009).

\section{Mental Models}

How do users even know which sources are present in an interaction? Human factors research has long examined how users' mental representations of how a system works as a way to predict behavior and design more usable systems (Wilson \& Rutherford, 1989). For example, computer users' mental models of computer viruses influence their security-related decisions (Wash, 2010). Mental models of a system are functional representations, and not necessarily complete or accurate cognitive diagrams of how a system actually works (Jones et al., 2011).

Mental models are related to source orientation because users may use mental models to identify cause and effect within an interaction with a computer. Mental models may include all the various sources in the interaction, or perhaps only some of them. And mental models may also include representations of relationships between sources and their capabilities, and these representations may affect how users orient themselves in the interaction.

Users' mental models may change over time, particularly as users develop expertise in using a system. This process of changing mental models may affect how users choose their orientation. Similarly, source orientation may affect mental models. Users who frequently change orientation and orient towards distant source regularly, may be able to better learn how those systems work and consequently form different mental models.

\section{Social Presence}

Social presence refers to "a sense of being with another person" (Biocca et al., 2003). Typical conceptual definitions of social presence in mediated environments have treated it as the degree to which an interaction is comparable to a real, face to face interaction (Steuer, 1992). This conceptual definition has been modified to include various motivational and psychological factors related to attention and involvement (Biocca et al., 2003; Riva, 2009). Social presence is determined both by the technological affordances of a medium (Steuer, 1992) (i.e. its media richness, the extent to which multiple cues are available for communication), and by individuals' motivation to treat an interaction as a social interaction, which may be the result of the context or simply of personality. Social presence is ultimately a measure of engagement towards social information. When a person is highly involved in a conversation or some other interaction, there is a high degree of social presence (even if the conversation is over IM). However, the salience of available information about others in a mediated environment can be antecedents to the level of engagement, making the relationship reciprocal in nature.

A sense of social presence strongly implies that a user is oriented towards the people with whom they communicate through computers. 


\section{SOURCE ORIENTATION MODEL}

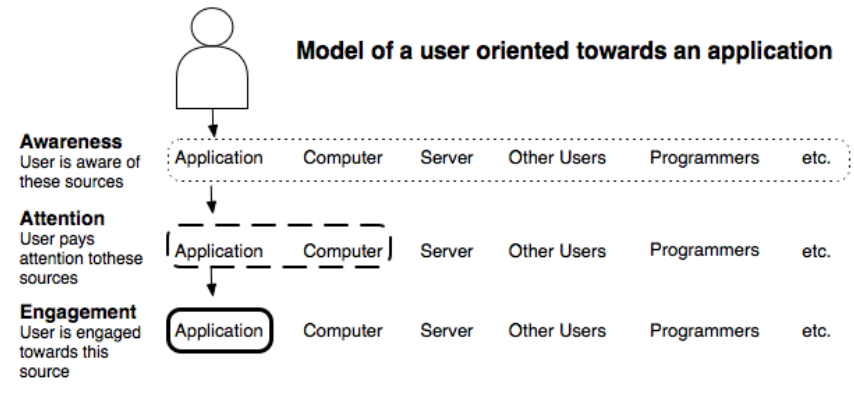

Figure 1

We have identified three conditions that must be met for a user to be oriented to a source, and illustrated this conceptualization of source orientation in Figure 1. These conditions are:

- Awareness- A user must be aware that the source exists and have some conceptualization of its role in the interaction. The source must be part of the user's mental model.

- Attention- A user must allocate attention to a source in order to be oriented towards that source. However, it is possible for a user to allocate some attention to multiple sources, so attention is not a sufficient condition for source orientation

- Engagement- A user must be actively engaged with a source and treat it as the locus of the interaction.

Understanding how users determine their source orientation at any given moment has important consequences for the design of user-centered computer systems. For this reason, we propose a theory of source orientation based on our review of related literatures. We will first describe the processes involved for each of the three conditions of source orientation. We will then synthesize these processes to describe more broadly how a user selects source orientation.

\section{Awareness}

Awareness comes from the user's mental model of the system they are using. For example, users must have a mental model of networked computing to be aware of the presence of remote servers, and without this awareness a user cannot be oriented to a remote server. Only users that have developed mental models that include remote sources such as servers, programmers, or organizations can be oriented towards these kinds of sources.

\section{Attention}

Stimuli in the environment can attract exogenous attention, whereas motivations and purposes for using a system may dictate endogenous attention. Interface and system designs may influence how users allocate attention during the interaction based on their relationship to the different attention processes. The user's goals when using a computer also allow him or her to allocate attention through top-down processing, and top-down processing is more capable of allocating attention to remote sources than bottomup processing because remote sources usually do not offer sensory stimuli.

\section{Engagement}

Engagement demands not only attention, but strong motivation and focus. In a video chat, for example, if a person is actively engaged or immersed in a conversation and is ignoring the computer that mediates the conversation, then this person's source orientation is towards the partner in the conversation. If oriented towards another person, users will sense a high degree of social presence. A user who is oriented towards the programmer of an application may be highly engaged as he or she tries to "get in their minds" and think about why certain programming decisions may have been made.

\section{Orientation and Re-orientation Factors}

We propose that some factors (called orientation factors) will tend to favor orientation towards immediate sources like the computer or software, and that other factors (re-orientation factors) will tend to favor remote or distant sources like programmers, organizations, or other users. Immediate sources require fewer resources for interaction than distant sources, and are therefore easier to engage in interaction and to maintain engagement. But re-orientation factors can trigger users to become engaged towards other sources. When the user re-orients it will typically be towards a more distant source. Figure 2 illustrates this concept. Below we have described these factors in greater detail.

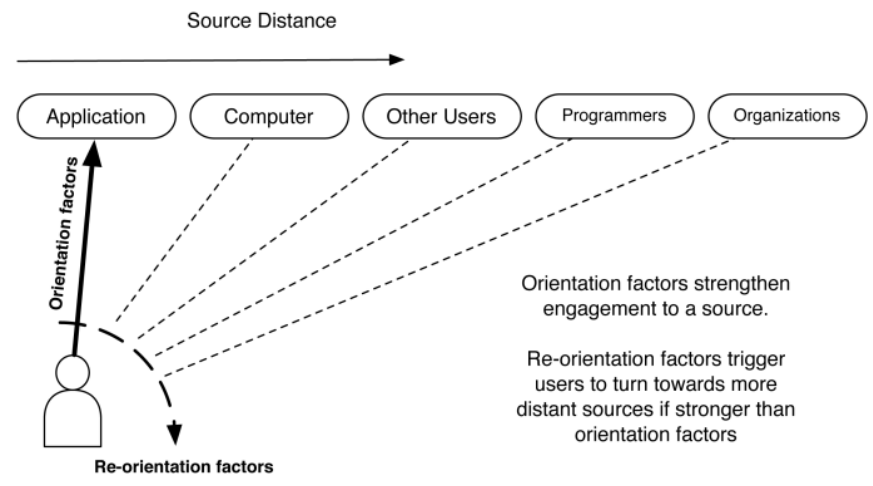

Figure 2

Orientation factors. Considerable work in computermediated communication has concluded that, despite technological advances, "distance matters" (Olson \& Olson, 2000). The mediation of communication degrades the quality of interactivity and accessibility between communicators. In $\mathrm{HCI}$, far more layers of mediation separate users from the programmers, organizations, content creators, policy makers etc. than from user interfaces and those with whom direct, two-way interaction is afforded. These less mediated sources can more easily attract users' exogenous attention processing (since the user can see or hear them directly), and the greater 
interactivity afforded by more immediate sources can enhance engagement.

CASA research provides some support that orientation towards an immediate source like the computer or application is somewhat of a "default" state for most users. Social responses to computers suggest high engagement with the computer, and CASA research (Tourangeau et al., 2003; Eckles et al., 2009; Shechtman \& Horowitz, 2003) has shown that when users are demonstrably not oriented towards the computer, they do not show social responses. For these reasons, our model suggests that users have an initial bias towards becoming oriented towards immediate sources such as the computer or application. We argue that one important reason for this default source orientation to the computer is simply its proximity. However, the concept of distance itself may be tied to users' mental models, and there may be variation among users regarding which sources are "closer."

The agency and direct interactivity of a source can also influence whether users become engaged and oriented towards that source. When users perceive that a source has some degree of agency and can act without direct input from some other source, users are likely to maintain orientation to that entity. In one study (Nowak \& Biocca, 2003), subjects treated an avatar in a 3-D environment the same when they were told it was controlled by a computer as when they were told it was controlled by a person, suggesting that the computer was able to demonstrate enough agency to maintain subjects' orientation. A difference in behavior would have suggested that subjects re-oriented towards a more distant source like the programmer or the researchers.

When a source has human-like appearance and agency, users are primed to treat it as human regardless of its true nature. The concept of social presence similarly suggests that people can become engaged towards entities in a virtual or mediated environment in a social manner as a result of the properties of the communication channel and their own personalities and motivations to have social interaction (Riva, 2009). Since some personality factors such as affiliative tendencies (Lee \& Jang, 2011) can enhance social presence, it is reasonable to assume that similar factors can strengthen orientation towards a source that is truly social in nature.

While interfaces using human-like appearance and behavior may prime user behavior through bottom-up processing of stimulus information, users still have goals and motivations to complete or fulfill when using computers. Therefore, the context in which a computer is used, or the task that is being performed, will undoubtedly have an influence on user engagement towards any source in the interaction. People generally follow the principle of least effort in accomplishing tasks or goals, which means that users are unlikely to change course in an interaction if their goals are being accomplished. Therefore, it is logical that goal satisfaction is an important engagement factor. As long as users are finding success in completing tasks or fulfilling motivations, it is unlikely that they will seek engagement with other sources.

Re-orientation factors. Any source has opportunities to capture user attention and cultivate engagement as long as users are aware of it. If engagement or re-orientation factors originate from sources other than a user's source orientation, there is naturally a possibility that the user will re-orient towards the new source.

Attribution theory discusses a number of biases that exist in the way humans attribute causes of behavior or outcomes. The negativity bias reported by Morewedge (2009) shows that when users experience negative outcomes, they are likely to attribute it to the agency of another person than to chance (even the chance of randomization by a computer program). In other words, when a user experiences a negative event, he or she may seek some agent to attribute blame. If their current source has not demonstrated enough agency to appropriately warrant blame, users may re-orient to a more distant source whose agency can be blamed. For example, if the power goes out while a computer user is working on some task, the user will likely find it inappropriate to blame the computer for its failure. Instead, they may seek a more distant source whose agency can more appropriately be blamed, such as the power company. This context of negative events suggests that disruption of user goals can trigger re-orientation.

In addition to negative events, unpredictable or inconsistent events and behaviors might also be able to trigger re-orientations. Takayama et al. (2009) found that when interacting with a robot, users prefer that the robots be agreeable with the user and that its voice come from its body. However, if the robot is not agreeable, people prefer that its voice be separated from its body. This is similar to many other studies of avatars and robots that show that consistency between behavioral and visual realism is more important than great fidelity in either dimension in terms of social responses as well as general affective responses (Garau et al., 2003; Nowak \& Biocca, 2003). This suggests that when there is inconsistency or unpredictability in the behavior of a source, a burden is placed on the user to reconcile that inconsistency. Since social responses to computers are limited when this inconsistency is present, it is plausible that users may have reoriented. This would be a logical strategy in many cases. If a computer's behavior is unpredictable, a strategy of "reverse engineering" is often useful. This strategy is a form of source orientation, as users try to get inside the minds of the system's designers rather than into the mind of the system itself.

\section{DISCUSSION}

Users' source orientation can impact their decisions and capabilities when interacting with computers. Therefore understanding how users become engaged towards some sources, while not being engaged towards others, is important for designing usable and human-centered technologies, particularly in the era of cloud and distributed computing.

We reviewed a broad set of literature that relates to this concept to develop a descriptive model. Our model argues that orientation towards immediate sources like the computer itself or applications is generally a default orientation, but that reorientation triggers such as malfunctions, explicit cues, or changes in motivation can lead users to change orientations and become engaged towards more distant sources such as programmers, servers, or the organization responsible for websites. 
This model, and a general understanding of source orientation, can assist designers and others to create more usable systems. The notion of designing a "user experience" into a system may hold an assumption that the user interface is the only important component in the interaction. However, if users are not oriented towards an immediate interface but instead to some other source, designers' intentions for the interactive experience may not be met. Here are a few examples of how source orientation can be applied to system design.

Security. Computer security or privacy decisions may depend on how users' source orientation. Users oriented towards their computers may be more vulnerable to phishing schemes or may be more likely to provide private data about themselves to large companies than users who are oriented towards those companies or towards the servers that carry information across the web. Warning messages in web browsers or other interfaces can provide explicit cues to encourage orienting themselves towards remote sources, at least briefly. If users spend time thinking about all sources that may have access to their data and consider their intentions, users will be able to make better security decisions online.

Troubleshooting. When computers malfunction, users' source orientation may affect their strategy for solving the problem. For example, if a website is loading slowly, there may be problems at many different places along the path from server to web browser. A user's source orientation may determine the strategy he or she takes to resolve the problem (e.g. restart the computer, reset network router, contact the website's customer support etc.). User support materials that provide information about how systems work and how problems can be corrected may do well to use anthropomorphic representations of remote sources such as servers or companies. This can help users develop engagement towards such sources and think through the potential role of these sources in causing the problem.

Recommender systems. As web and mobile technologies increasingly become context-aware and provide recommendations to users about decisions, users' source orientation may affect how recommendations are received and used in a decision. A user oriented towards her phone may find a notification that she is near a highly-rated restaurant helpful. But if she is oriented towards the company that makes the phone, she may find this to be an obtrusive advertisement. Or, she may feel the notification provides some engagement towards others in her community who have rated the restaurant highly, and may therefore be accepting of the recommendation. Designers of recommender systems and similar systems should consider which source orientation would help users make desired decisions. They should then consider how to induce orientation towards that source and how to prevent events that may trigger re-orientation.

We have provided here a theory-based description of source orientation and a systematic model that describes how users determine source orientation at any given moment. We feel this model can be valuable for system designers striving to create usable and human-centered computing systems. We also feel this concept demands further work from human factors researchers so that precise predictions can be made about user behavior and concrete design recommendations can be made that account for source orientation in computer systems.

\section{References}

Ashcraft, M. H., \& Ashcraft, M. H. (2006). Cognition. Upper Saddle River, N.J: Pearson Prentice Hall.

Biocca, F., Harms, C., \& Burgoon, J. K. (2003). Toward a more robust theory and measure of social presence: Review and suggested criteria. Presence: Teleoperators and virtual environments, 12(5), 456-480.

Chabris, C. F., \& Simons, D. J. (2010). The invisible gorilla: And other ways our intuitions deceive us. New York: Crown.

Eckles, D., Wightman, D., Carlson, C., Thamrongrattanarit, A., Bastea-Forte, M., \& Fogg, B. J. (2009, April). Social responses in mobile messaging: influence strategies, self-disclosure, and source orientation. In Proceedings of the SIGCHI Conference on Human Factors in Computing Systems (pp. 1651-1654). ACM

Garau, M., Slater, M., Vinayagamoorthy, V., Brogni, A., Steed, A., \& Sasse, M. A. (2003, April). The impact of avatar realism and eye gaze control on perceived quality of communication in a shared immersive virtual environment. In Proceedings of the SIGCHI conference on Human factors in computing systems (pp. 529-536). ACM.

Gilbert, D. T., \& Malone, P. S. (1995). The correspondence bias. Psychological bulletin, 117(1), 21.

Hoffmann, L., Krämer, N. C., Lam-Chi, A., \& Kopp, S. (2009). Media equation revisited: do users show polite reactions towards an embodied agent?. In Intelligent Virtual Agents (pp. 159-165). Springer Berlin Heidelberg.

Jones, N. A., Ross, H., Lynam, T., Perez, P., \& Leitch, A. (2011). Mental Models: An Interdisciplinary Synthesis of Theory and Methods. Ecology \& Society, 16(1).

Kelley, H. H., \& Michela, J. L. (1980). Attribution theory and research. Annual review of psychology, 31(1), 457-501.

Lee, E. J., \& Jang, J. W. (2013). Not So Imaginary Interpersonal Contact With Public Figures on Social Network Sites How Affiliative Tendency Moderates Its Effects. Communication Research, 40(1), 27-51.

Morewedge, C. K. (2009). Negativity bias in attribution of external agency. Journal of Experimental Psychology: General, 138(4), 535.

Nass, C., \& Moon, Y. (2000). Machines and mindlessness: Social responses to computers. Journal of social issues, 56(1), 81-103.

Olson, G. M., \& Olson, J. S. (2000). Distance matters. Human-computer interaction, 15(2), 139-178.

Nowak, K. L., \& Biocca, F. (2003). The effect of the agency and anthropomorphism on users' sense of telepresence, copresence, and social presence in virtual environments. Presence: Teleoperators and Virtual Environments, 12(5), 481-494.

Riva, G. (2009). Is presence a technology issue? Some insights from cognitive sciences. Virtual Reality, 13(3), 159-169.

Shechtman, N., \& Horowitz, L. M. (2003). Media inequality in conversation: how people behave differently when interacting with computers and people. In Proceedings of the SIGCHI conference on Human factors in computing systems (pp. 281-288). ACM.

Steuer, J. (1992). Defining virtual reality: Dimensions determining telepresence. Journal of Communication, 42(4), 73-93.

Sundar, S. S., \& Nass, C. (2000). Source orientation in human-computer interaction: Programmer, networker, or independent social actor. Communication Research, 27(6), 683-703.

Takayama, L., Groom, V., \& Nass, C. (2009, April). I'm sorry, Dave: I'm afraid I won't do that: Social aspects of human-agent conflict. In Proceedings of the SIGCHI Conference on Human Factors in Computing Systems (pp. 2099-2108). ACM.

Tourangeau, R., Couper, M. P., \& Steiger, D. M. (2003). Humanizing selfadministered surveys: experiments on social presence in web and IVR surveys. Computers in Human Behavior, 19(1), 1-24.

Wash, R. (2010). Folk models of home computer security. In Proceedings of the Sixth Symposium on Usable Privacy and Security (p. 11). ACM.

Wilson, J. R., \& Rutherford, A. (1989). Mental models: Theory and application in human factors. Human Factors: The Journal of the Human Factors and Ergonomics Society, 31(6), 617-634. 